\title{
Investigating non-uniform scaling behaviour in temporal fluctuations of seismicity
}

\author{
L. Telesca and M. Lovallo \\ Dipartimento Infrastrutture e Mobilità, Ufficio Difesa del Suolo, Regione Basilicata, Potenza, Italy \\ Received: 30 June 2008 - Revised: 28 July 2008 - Accepted: 28 July 2008 - Published: 1 September 2008
}

\begin{abstract}
Scaling behaviour in nonstationary time series can be successfully detected using the detrended fluctuation analysis (DFA). Observational time series often do not show a stable and uniform scaling behaviour, given by the presence of a unique clear scaling region. The deviations from uniform power-law scaling, which suggest the presence of changing dynamics in the system under study, can be identified and quantified using an appropriate instability index. In this framework, the scaling behaviour of the 1981-2007 seismicity in Umbria-Marche (central Italy), which is one of the most seismically active areas in Italy, was investigated. Significant deviations from uniform power-law scaling in the seismic temporal fluctuations were revealed mostly linked with the occurrence of rather large earthquakes or seismic clusters.
\end{abstract}

\section{Introduction}

The complexity of the dynamics of geophysical processes is often characterized by self-similarity, which suggests that their dynamics may be interpreted as due to many components interacting over a wide range of time or space scales (Ashkenazy et al., 2003). A typical example of complex systems are earthquakes, characterized by power-law distribution of spatial, temporal and energy parameters. The existence of power-laws also qualifies earthquakes as a good example of fractal systems.

The interevent intervals (times between two successive seismic events) follow an exponential decreasing distribution (Poissonian) for completely random sequences, while they are generally power-law distributed for time-clusterized sequences (Telesca et al., 2001). But the probability density

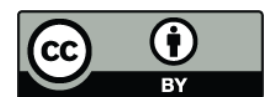

Correspondence to: L. Telesca (luciano.telesca@imaa.cnr.it) function of the interevent intervals is only one feature of the process and it reveals none about the correlation properties (Turcott et al., 1994). Therefore, time-fractal second-order methods are necessary to investigate the temporal fluctuations of seismic sequences more deeply. The use of statistics like the Allan Factor (Allan, 1966), the Fano Factor (Lowen and Teich, 1995), the Detrended Fluctuation Analysis (DFA) (Peng et al., 1995), has allowed getting more insight into the time dynamics of seismicity. All these measures lead to the determination of a scaling exponent, estimated by a linear fitting procedure performed on the power-law statistics plotted in log-log scales. Such scaling exponent informs about the dynamical properties of an earthquake sequence, in terms of its correlation structures and its memory phenomena and several studies have been performed aiming at the: (i) discrimination between Poissonian and clusterized sequences, (ii) spatial variability of time-clustering behaviour, and (iii) magnitude-variability of the property of time-clusterization (Telesca et al., 2003). Furthermore, the numerical value of the scaling exponent allows to determine quantitatively the strength of the temporal fluctuations of seismicity.

In the present paper, the scaling behaviour of the series of earthquakes occurred in Umbria-Marche, central Italy (Fig. 1) was performed. This area was struck by a strong earthquake occurred on 26 September 1997, with a duration magnitude of 5.8 (this event will be indicated as EQ0 hereafter). After that, the Umbria-Marche region, central Italy, was deeply studied and many investigations have been performed regarding its geodynamical features (Boncio and Lavecchia, 2000; Calamita et al., 2000), active tectonics (Galadini et al., 1999; Cinti et al., 2000), spatio-temporal seismic distribution (Ripepe et al., 2000; Di Giovambattista and Tyupkin, 2000), induced geophysical effects (Esposito et al., 2000). This area is characterized by a welldocumented historical and instrumental seismicity, mainly confined within the upper part of the crust $(<16 \mathrm{~km})$ (Lavecchia et al., 1994).

Published by Copernicus Publications on behalf of the European Geosciences Union. 


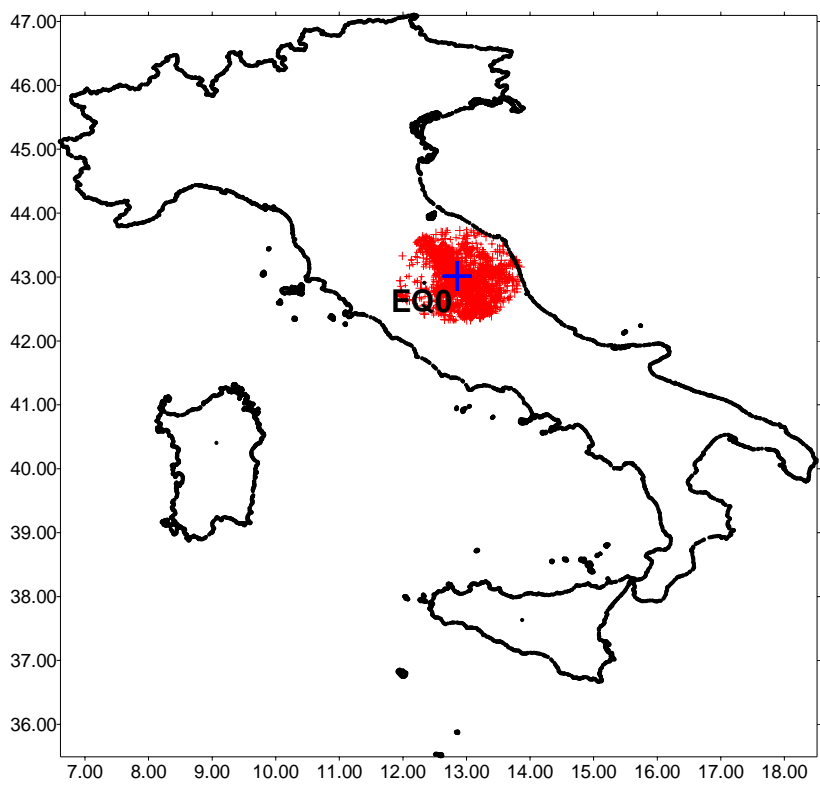

Fig. 1. Spatial distribution of seismicity in Umbria-Marche area from 1981 to 2007.

\section{The method}

A series of earthquakes can be represented by a temporal point process, that describes events that occur at some random locations in time (Cox and Isham, 1980), and it can be expressed by a finite sum of Dirac's delta functions centered on the occurrence times $t_{i}$, with amplitude $A_{i}$ proportional to the magnitude of the earthquake:

$y(t)=\sum_{i=1}^{N+1} A_{i} \delta\left(t-t_{i}\right)$

$(N+1)$ represents the number of events recorded. This process can be described by the set of the interevent times, that is the series of the time intervals between two successive earthquakes.

The DFA was proposed by Peng et al. (1995), and it avoids spurious detection of correlations that are artifacts of nonstationarity, that often affects experimental data. Such trends have to be well distinguished from the intrinsic fluctuations of the system in order to find the correct scaling behaviour of the fluctuations. Very often we do not know the reasons for underlying trends in collected data and we do not know the scales of underlying trends. DFA is a method for determining the scaling behaviour of data in the presence of possible trends without knowing their origin and shape.

The methodology operates on the series of the interevent times $\tau_{i}$, where $i=1,2, \ldots, N$ and $N$ is the length of the series. With $\bar{\tau}$ we indicate the mean interevent time.
The series is first integrated

$y(k)=\sum_{i=1}^{k}\left[\tau_{i}-\bar{\tau}\right]$

with $k=1 . . N$.

Next, the integrated time series is divided into boxes of equal length, $n$. In each box a least-squares polynomial $y_{n}(k)$ of degree $p$ is fit to the data, representing the trend or order $p$ in that box. Next we detrend the integrated time series $y(k)$ by subtracting the local trend $y_{n}(k)$ in each box. The rootmean-square fluctuation of this integrated and detrended time series is calculated by

$F(n)=\sqrt{\frac{1}{N} \sum_{k=1}^{N}\left[y(k)-y_{n}(k)\right]^{2}}$.

Repeating this calculation over all box sizes, we obtain a relationship between $F(n)$, that represents the average fluctuation as a function of box size, and the box size $n$. If $F(n)$ behaves as a power-law function of $n$, data present scaling:

$F(n) \propto n^{d}$.

Under these conditions the fluctuations can be described by the scaling exponent $d$, representing the slope of the line fitting $\log F(n)$ to $\log n$. The estimate of the $d$-value, performed by a least square method, furnishes information about the type of correlations in the interevent series; furthermore, it quantifies the growth of the root mean square fluctuations $F(n)$. For an uncorrelated seismic sequence, $d=0.5$. If there are only short-range correlations, the initial slope may be different from 0.5 but will approach 0.5 for large window sizes $n . d>0.5$ indicates the presence of persistent long-range correlations, meaning that a large (compared to the average) interevent interval is more likely to be followed by large one and vice versa. $d<0.5$ indicates the presence of antipersistent long-range correlations, meaning that a large (compared to the average) interevent interval is more likely to be followed by small one and vice versa. $d=1$ indicates flicker-noise dynamics, typical of systems in a self-organized critical state. $d=1.5$ characterizes processes with Brownian-like dynamics.

\section{Results and discussion}

We investigated the interevent time series of earthquakes occurred in Umbria-Marche area, central Italy, from 1981 to 2007. We considered the earthquakes $(M \geq 2.1)$ whose epicenters were located within a $80 \mathrm{~km}$-radius circular area centered on the epicenter of the strongest event of the series (EQ0) (Fig. 1). The seismicity data were extracted from the INGV catalogue, publicly available on http://www.ingv.it. The choice of such selection was given by the great seismological importance given by this event, and by the high activity characterizing such seismic area, featured by several rather large events during the investigation period. 
To examine transient phenomena, the seismic interevent time series was analysed in overlapping windows of fixed number of events. Therefore the DFA method was performed in a sliding window of 300 events, in order to have a sufficient amount of data. The shift between two successive windows was set to one event, in order to have a good smoothing among the calculated values. In each window, we applied the DFA, removing the polynomial trends up to the 4 th order. Furthermore, after calculating the fluctuation curve $\log [F(n)] \sim \log (n)$, using the DFA method, in each window we calculated the scaling instability index $\beta$, as follows: the local scaling exponent $\chi(n)$ can be defined as the local derivative (slope) of the fluctuation function $\log [F(n)] \sim \log (n)$,

$\chi(n) \equiv d[\log (F(n)] / d[\log (n)]$.

If $\chi(n)$ is constant for different timescales $n$, the scaling is stable. Substantial variation of $\chi(n)$ with $\mathrm{n}$ indicates that the scaling behaviour is unstable and deviates from the uniform power-law (Viswanathan et al., 1997). To quantitatively measure the deviation from stable power-law scaling, the scaling instability index $\beta$ can be defined as the standard deviation of the local scaling exponents $\chi(n)$, in the timescales of interest. High values of the index $\beta$ indicate large deviations from uniform power-law. In some cases the high value of the instability index is also given by the presence of different stable power-law regimes, characterized each of them by well defined scaling exponent. Using this definition of scaling instability index, each calculated $\beta$ value was associated with the time of occurrence of the last event in the window. Figure 2 shows the time variation of $\beta$. It is also shown the dotted line $\mu+2 \sigma$, which can be considered like the threshold for a $\beta$ value to be taken as anomalous. Several features are revealed by this analysis: i) the variation of the index $\beta$ is characterized by a spiky behaviour, where the highest values denote increase of instability; ii) three clusters of events with magnitude $M>4$ (which can be considered as a relatively high magnitude) were identified in the seismicity of the area, and are indicated in Fig. 2 by vertical red arrows;

iii) in correspondence with these clusters the instability in$\operatorname{dex} \beta$ shows an increasing spiky behaviour indicated by letters a to $\mathrm{d}$; the anomalous $\beta$ values indicated by letters a, $\mathrm{c}$ and $\mathrm{d}$ are clearly linked with the earthquakes; the spike $\mathrm{b}$ could be connected with the aftershock activity following the strong earthquake EQ0.

These results evidence that rather large events are correlated with an anomalous increase of the instability index, which indicates that the time-scaling behaviour of the seismicity, given by the power-law shape of the fluctuation function $F(n)$, becomes unstable. The occurrence of an intense seismic event contributes to break the stability of the scaling behaviour of the background seismicity, mainly given by the smaller events. The rupture of the scaling stability indicates that the earthquake distribution is like to be "disturbed" by the occurrence of a large event. Such result seems to confirm

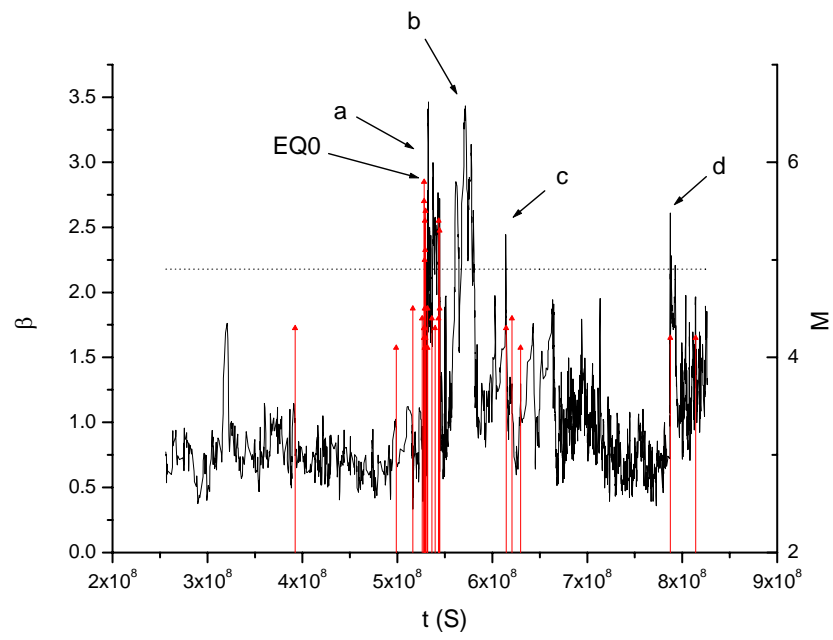

Fig. 2. Time variation of $\beta$ for the seismicity data plotted in Fig. 1. The values of $\beta$ were calculated in a window of 300 events shifting through the entire catalogue by one event. Each value was associated to the time of occurrence of the last event in the window. The red vertical arrows indicate the seismic events with magnitude $M>4$.

what has been obtained in a rock fracture experiment with drive control (Kuksenko et al., 2007). In fact, it was found that at the initial stage, the events occur predominantly in certain "weak points" whose distribution is mainly determined by the structural heterogeneity and mechanical prehistory of samples. The system is dynamically connected through elastic interactions and oscillation processes and its scaling behaviour shows its critical state that could be maintained as long as the structural rearrangements of the given scale level would enable to compensate the energy release. The appearance of larger-scale events (primary damages of upper hierarchic level) reduces the contribution of smaller-scale events to the formation of the fault geometry; and in this period of hierarchic transition the scaling properties of the system become disturbed due to low occurrence of large events that determine the further evolution of the system

\section{Conclusions}

The detrended fluctuation analysis of seismicity data observed in Umbria-Marche area, central Italy from 1981 to 2007, reveals scaling behaviour in the temporal dynamics of the seismic interevent time series. The instability parameter $\beta$ identifies and quantifies the deviations from uniform power-law scaling. Its time variation reveals a significant increase connected with the occurrence of the larger earthquakes.

Edited by: P. F. Biagi

Reviewed by: M. Rouai and another anonymous referee 


\section{References}

Allan, D. W.: Statistics of Atomic Frequency Standards, Proc. IEEE, 54, 221-230, 1966.

Ashkenazy, Y., Havlin, S., Ivanov, P. Ch., Peng, C.-K., SchulteFrohlinde, V., and Stanley, H. E.: Magnitude and sign scaling in power-law correlated time series, Physica A, 323, 19-41, 2003.

Boncio, P. and Lavecchia, G.: A structural model for active extension in Central Italy, J. Geodyn., 29, 233-244, 2000.

Calamita, F., Coltorti, M., Piccinini, D., Pierantoni, P. P., Pizzi, A., Ripepe, M., Scisciani, V., and Turco, E.: Quaternary faults and seismicity in the Umbro-Marchean Apennines (Central Italy): evidence from the 1997 Colfiorito earthquake, J. Geodyn., 29, 245-264, 2000.

Cinti, F. R., Cucci, L., Marra, F., and Montone, P.: The 1997 Umbria-Marche earthquakes (Italy): relation between the surface tectonic breaks and the area of deformation, J. Seismol., 4, 333343, 2000.

Cox, D. R. and Isham, V.: Point Processes, Chapman and Hall, London, 1980.

Di Giovambattista, R. and Tyupkin, Y. S.: Spatial and temporal distribution of seismicity before the Umbria-Marche September 26, 1997 earthquakes, J. Seismol., 4, 589-598, 2000.

Esposito, E., Porfido, S., Simonelli, A. L., Mastrolorenzo, G., and Iaccarino, G.: Landslides and other surface effects induced by the 1997 Umbria-Marche seismic sequence, Eng. Geol., 58, 353376, 2000.

Galadini, F., Galli, P., Leschiutta, I., Monachesi, G., and Stucchi, M.: Active tectonics and seismicity in the area of the 1997 earthquake sequence in central Italy: A short review, J. Seismol., 3, 167-175, 1999.
Kuksenko, V., Tomilin, N., and Chmel, A.: The rock fracture experiment with a drive control: A spatial aspect, Tectonophysics, 431, 123-129, 2007.

Lavecchia, G., Brozzetti, F., Barchi, M., Menichetti, M., and Keller, J. V. A.: Seismotectonic zoning in east-central Italy deduced from an analysis of the Neogene to Present deformations and related stress fields, Geol. Soc. Am. Bull., 106, 1107-1120, 1994.

Lowen, S. B. and Teich, M. C.: Estimation and Simulation of Fractal Stochastic Point Processes, Fractals, 3, 183-210, 1995.

Peng, C.-K., Havlin, S., Stanley, H. E., and Goldberger, A. L.: Quantification of scaling exponents and crossover phenomena in nonstationary heartbeat time series, Chaos, 5, 82-87, 1995.

Ripepe, M., Piccinini, D., and Chiaraluce, L.: Foreshock sequence of September 26th, 1997 Umbria-Marche earthquakes, J. Seismol., 4, 387-399, 2000.

Telesca, L., Lapenna, V., and Macchiato, M.: Spatial variability of time-correlated behaviour in Italian seismicity, Earth Planet. Sci. Lett., 212, 279-290, 2003.

Telesca, L., Cuomo, V., Lapenna, V., and Macchiato, M.: Statistical analysis of fractal properties of point processes modeling seismic sequences, Phys. Earth Planet. Int., 125, 65-83, 2001.

Turcott, R. G., Lowen, S. B., Li, E., Johnson, D. H., Tsuchitani, C., and Teich, M. C.: A Nonstationary Poisson Point Process Describes the Sequence of Action Potentials Over Long Time Scales in Lateral-Superior-Olive Auditory Neurons, Biol. Cybern., 70, 209-217, 1994.

Viswanathan, G. M., Peng, C.-K., Stanley, H. E., and Goldberger, A. L.: Deviations from uniform power law scaling in nonstationary time series, Phys. Rev. E, 55, 845-849, 1997. 